Garcés Ayerbe, Rivera Torres, Murillo Luna(2014) / Revista de Empresa Familiar, 4(1), 59-71.

\title{
Inversión medioambiental y resultado financiero en las empresas familiares españolas
}

\section{Environmental investment and financial performance in Spanish family business}

\author{
Concepción Garcés Ayerbe $^{\mathrm{a}} \cdot$ Pilar Rivera Torres $^{\mathrm{b}} \cdot \mathrm{J}_{\text {osefina Lucía Murillo Luna }}{ }^{\mathrm{*}}$ \\ ${ }^{a}$ Departamento de Dirección y Organización de Empresas. Universidad de Zaragoza (Zaragoza) \\ ${ }^{b}$ Departamento de Dirección de Marketing e Investigación de Mercados. Universidad de Zaragoza (Zaragoza)
}

\section{A T OS A R TÍCULO}

Historial:

Recibido 04-09-2013

Aceptado 05-04-2014

Palabras clave:

Inversión Medioambiental

Resultado Financiero

Tipo de propiedad

Códigos JEL:

C12, M14

\section{A R T I C LE IN F O}

Article history:

Received 04-09-2013

Accepted 05-04-2014

Keywords:

Environmental Investment

Financial performance

Ownership structure

JEL codes:

C12, M14

\begin{abstract}
R E S U M E N
Ante la falta de consenso sobre la relación entre los resultados medioambientales y los resultados económicos de las empresas, y las aportaciones de diferentes autores que explican esta controversia por la existencia de ciertos condicionantes, el presente trabajo pretende analizar el posible efecto moderador de la forma de gobierno de la empresa en esta relación. Los resultados del análisis de regresión de una muestra de 1916 empresas del sector manufacturero español indican que, en efecto, la situación win-win en la que inversión medioambiental implica una mejora de los resultados medioambientales y económicos no puede darse por garantizada $\mathrm{y}$, además, el impacto de la inversión medioambiental en los resultados financieros es mayor en el caso de las empresas pequeñas de tipo familiar.
\end{abstract}

\footnotetext{
* Autor de contacto.

Correoselectrónicos: cgarces@unizar.es,privera@unizar.es,jmurillo@unizar.es
}

El presente trabajo forma parte de los resultados obtenidos bajo el marco del proyecto de investigación ECO2010-21393-C04-04 financiado por MICINN-FEDER, el proyecto de investigación ECO2012-36290-C03-01 financiado por MINECO y el Grupo de Investigación de Excelencia CREVALOR reconocido por la DGA y financiado por FSE. Las autoras también agradecen la financiación recibida de la Cátedra de Empresa Familiar de la Universidad de Zaragoza. 


\section{Introducción}

La relación entre los resultados medioambientales y los resultados económicos de las empresas ha sido extensamente estudiada en la literatura desde los años setenta. Sin embargo, existe todavía cierta controversia acerca del signo positivo versus negativo- de esta relación (Griffin y Mahon, 1997; Margolis y Walsh, 2003). En efecto, aunque son cada vez más numerosos los trabajos que, bajo el lema "It Pays to be Green", argumentan, y respaldan con evidencia empírica en algunos casos, que el esfuerzo medioambiental de las empresas se traduce en una mejora de sus resultados financieros (Porter, 1991; Porter y Van der Linde, 1995; Hart, 1995; Hart y Ahuja, 1996; Russo y Fouts, 1997; King y Lenox, 2001; Clemens, 2006; Guenster et al, 2006; Walls et al., 2008), todavía recientes investigaciones empíricas encuentran una relación negativa o no significativa en el estudio de esta relación (e.g. Filbeck y Gorman, 2004, Telle, 2006; Ziegler et al., 2007 o Cañón-de-Francia y Garcés-Ayerbe, 2009).

Esta falta de consenso ha sido justificada en la literatura de diversas formas. Filbeck y Gorman (2004), por ejemplo, advierten que en el estudio de la relación entre los resultados medioambientales y los resultados económicos el carácter más o menos estricto de los requerimientos medioambientales juega un importante papel. En coherencia con este argumento, King y Lenox $(2001 ; 2002)$ o Guenter et al. (2006) consideran que la relación entre los resultados medioambientales y financieros está condicionada por la forma en las que las medidas de tipo medioambiental son implementadas. Más concretamente, King y Lenox (2002) encuentran fuerte evidencia de que los mecanismos de protección medioambiental basados en la prevención de la contaminación dan lugar a mejoras en el rendimiento financiero pero los basados en la corrección de la contaminación a través de la adopción de medidas al final del proceso productivo no afectan al resultado financiero. En efecto, tal y como hacen notar Guenster et al. (2006), la información financiera que contiene el resultado medioambiental no es evidente por sí misma. Estos autores, de acuerdo con otros académicos como Hart y Ahuja (1996), King y Lenox (2002) o Russo y Fouts (1997) enfatizan que las empresas pueden alcanzar resultados medioambientales a través de mecanismos de corrección de la contaminación soluciones reactivas- pero que la probabilidad de mejorar la eficiencia y la rentabilidad es menor que en el caso de implementar técnicas proactivas de prevención de la contaminación.
Las conclusiones que se derivan de estos trabajos están conduciendo las recientes investigaciones acerca de la relación entre los resultados medioambientales y los resultados económicos hacia el estudio de cómo las condiciones en las que se lleva a cabo la implementación de sistemas de gestión medioambiental pueden condicionar esta relación. En este sentido, King y Lenox (2001) argumentan que "When does it pay to be green? podría ser una cuestión más relevante que "Does it pay to be green?".

Es aquí donde encuentra sentido la presente investigación, que pretende analizar la repercusión de las inversiones medioambientales en los resultados financieros de las empresas considerando el efecto moderador en dicha relación de la forma de gobierno de la empresa, distinguiendo en este caso entre empresas familiares y no familiares.

Existen algunos trabajos en la literatura que han analizado cómo la condición de empresa familiar versus no familiar ayuda a explicar el resultado medioambiental de las empresas. Sin embargo, en lo que alcanzamos a ver, no existen trabajos previos en la literatura que consideren este aspecto como moderador de la relación entre los resultados medioambientales y los resultados económicos. La consideración de esta variable resulta de gran interés. Más aún, de acuerdo con la literatura sobre empresa familiar, ignorar esta variable en la investigación organizacional conduce a modelos teóricos erróneamente especificados y a resultados empíricos no aplicables a la mayoría de las organizaciones empresariales (Sharma y Sharma, 2011). Así pues, la consecución de los objetivos planteados en este proyecto permitiría, por tanto, aportar resultados novedosos al estado de conocimiento sobre el tópico.

La estructura del trabajo es la siguiente: en primer lugar se realiza una revisión de la literatura previa y se plantean las hipótesis. Posteriormente se realiza un análisis empírico que permite comprobar si se confirman las hipótesis planteadas. Por último, se exponen los resultados del análisis empírico y se presentan las principales conclusiones del estudio.

\section{Relación entre resultados medioambientales y resultados económicos}

Dentro del marco teórico de la Visión Basada en los Recursos Naturales y del Enfoque de las Capacidades Dinámicas, existe cierto consenso en la literatura, bajo la denominada "Hipótesis Porter", acerca de que las estrategias medioambientales 
proactivas se basan en recursos clave y generan capacidades que dan lugar a una situación win-win en la que tanto los resultados medioambientales como los económicos pueden ser mejorados.

En uno de los artículos originarios de esta línea de pensamiento, Porter (1991) se muestra en desacuerdo con la creencia, predominante durante la década de los años ochenta, de que la regulación medioambiental perjudica la competitividad de las empresas. De acuerdo con este autor, el conflicto entre la protección medioambiental y la competitividad económica es una falsa dicotomía. Si los estándares medioambientales están adecuadamente diseñados por los reguladores, indican Porter y van der Linde (1995), su cumplimiento orientará a las empresas hacia la innovación y hacia la búsqueda de formas más eficientes de producción. Además, las empresas afectadas por la regulación medioambiental se ven obligadas a reconsiderar sus procesos de producción, lo que facilita la detección de ineficiencias $y$ promueve la mejora continua. La sustitución de los equipos de producción por otros más modernos y basados en mínimo consumo en las empresas sometidas a regulación medioambiental aumentará la productividad de las mismas, al mismo tiempo que se reduce el daño medioambiental (Xepapadeas y Zeeuw, 1999).

Otro de los autores más citados dentro de esta línea de pensamiento es Hart (1995), quien sostiene que la ventaja competitiva de las empresas deberá ser asentada cada vez más en capacidades y recursos clave que faciliten una actividad económica medioambientalmente sostenible. Y concentra sus argumentos en las capacidades "prevención de la polución", "gestión de los productos" y "desarrollo sostenible", que facilitan ventajas competitivas a través de la reducción de costes, la anticipación a los competidores y la mejora de las relaciones con los stakeholders.

Los argumentos originariamente establecidos por Porter (1991); Hart (1995), Porter y van del Linde (1995) o Xepapadeas y Zeeuw (1999), entre otros, han sido posteriormente secundados y completados en un buen número de trabajos (Sharma y Vredenburg, 1998; Christmann, 2000; AragónCorrea y Sharma, 2003; Clemens y Bakstran, 2010). Existe, además, abundante evidencia empírica que apunta hacia una relación positiva entre los resultados medioambientales y los resultados económicos de las empresas (por ejemplo, en Hart y Auja, 1996; Russo y Fouts, 1997; Sharma y Vredenburg, 1998; Judge y Douglas, 1998; Klassen y Whybark, 1999; Konar y Cohen, 2001; Telle,
2006; González-Benito y González-Benito, 2005a; Darnall et al., 2007; Bosworth y Clemens, 2011; Nakamura, 2011).

Sin embargo, existen también otros trabajos cuyos resultados no apoyan estos argumentos. Los primeros en cuestionar la naturaleza de la relación entre la rentabilidad económica y los resultados medioambientales son King y Lenox (2001), planteando la posible existencia de ciertos atributos inherentes a la empresa que pudieran explicar el signo positivo de esta relación. Posteriormente, autores como Filbeck y Gorman (2003), Elsayed y Paton (2004), Telle (2006), Ziegler et al. (2007), Cañón-de-Francia y Garcés-Ayerbe (2009) o, más recientemente, Lanoie et al. (2011), continúan sugiriendo la necesidad de profundizar en el análisis de esta relación. Estos trabajos se acompañan de estudios empíricos en los que se exploran estas cuestiones con metodologías diversas, coincidiendo todos ellos en lo prematuro de afirmar la existencia de una relación positiva y llegando a la conclusión de que, más que preguntarse sobre la rentabilidad económica de los resultados medioambientales, lo importante es conocer las condiciones en las que se da esta relación positiva.

Los argumentos y evidencia empírica encontrados en la literatura nos conducen a cuestionar la existencia -o no- de una posible relación lineal entre los beneficios económicos y la inversión medioambiental, estableciendo la siguiente hipótesis:

H1: Existe una relación lineal entre la inversión medioambiental privada y los beneficios económicos de las empresas.

\section{Empresa familiar y relación entre resultados medioambientales $y$ resultados económicos: antecedentes}

Corona y Telléz (2011) definen empresa familiar como aquélla en la que un grupo familiar está en condiciones de designar al máximo ejecutivo de la compañía y de fijar la estrategia empresarial de la misma, y todo ello con el objetivo de continuidad generacional, basado en el deseo conjunto de fundadores y sucesores de mantener el control de la propiedad y la gestión en la familia. Así entendidas, las empresas familiares desempeñan un papel importante en la realidad económica. De acuerdo con los datos aportados por Gallego (2012), las empresas familiares suponen el $85 \%$ del tejido empresarial español, representan el $50 \%$ de las empresas que cotizan en bolsa, generan el 75\% del 
empleo privado, realizan el $59 \%$ de las exportaciones y su facturación equivale al $70 \%$ del PIB español.

La relevancia de la empresa familiar en la economía ha suscitado una gran cantidad de trabajos de investigación teóricos y empíricos que tratan de analizar sus características idiosincrásicas. En este trabajo tratamos de contribuir en el estudio de las características particulares de las empresas familiares, a través del análisis de la relación entre los resultados medioambientales y los resultados económicos de dichas empresas.

La literatura que analiza las características distintivas de las empresas familiares en materia de protección medioambiental es reciente. De la revisión de esta literatura se concluye que existen argumentos teóricos y evidencia empírica que respaldan la idea de que las empresas familiares tienen un comportamiento en materia medioambiental que difiere del de las no familiares. Berrone et al. (2009), por ejemplo, ponen de manifiesto que las empresas familiares son más proclives a seguir una política medioambiental. Los motivos por los que, según estos autores, las empresas familiares se preocupan especialmente por la protección medioambiental son: en primer lugar porque quieren evitar ser estigmatizadas por los ciudadanos como irresponsables, en segundo lugar porque las empresas familiares son más sensibles a las necesidades y presiones de la comunidad en materia medioambiental $\mathrm{y}$, en tercer lugar, porque las empresas familiares tienden a depender en menor grado de los incentivos financieros a largo plazo como mecanismo para promover la responsabilidad medioambiental.

Otros autores, como Huang et al. (2009), ponen de manifiesto que la actitud medioambiental de las empresas familiares es también diferente desde el punto de vista de las relaciones con los stakeholders. Concretamente estos autores demuestran que la relación entre la presión de los stakeholders internos y la adopción de innovación medioambiental es mayor en las empresas familiares que en las no familiares. Siguiendo con la relación entre la forma de gestión de los stakeholders y la actitud medioambiental de la empresa, Uhlaner et al. (2004), ponen de manifiesto que en las empresas familiares hay una relación especial con los stakeholders, concretamente con los clientes, que favorece la adopción de sistemas de gestión medioambiental.

Bingham et al. (2011), argumentan también que las empresas familiares tienden a comprometerse más en términos de Responsabilidad Social Corporativa que las no familiares y demuestran que las empresas con mayor participación del fundador de la empresa familiar tiene más iniciativas sociales con comunidades, empleados y clientes. En la misma línea de argumentación, Wagner (2010), concluye que las empresas familiares están más a favor de la protección del medio ambiente como parte de su responsabilidad social corporativa. En éste último artículo el autor demuestra que el hecho de ser empresa familiar proporciona un efecto moderador positivo a la relación entre responsabilidad social corporativa y la innovación, con elevados beneficios sociales.

Adicionalmente, autores como Craig y Dibrell (2006) concluyen que la política de medio ambiente influye más en la innovación empresarial en las empresas familiares, y menos en las no familiares. Estos autores demuestran también que la política de protección del medio ambiente determina unos mejores resultados en el caso de empresas de tipo familiar en comparación con empresas no familiares, datos fuertemente contrastados en su estudio.

De los trabajos anteriores se deduce que las empresas familiares tienden a adoptar estrategias medioambientales más proactivas, más ajustadas a las necesidades concretas de los stakeholders, más basadas en la innovación $\mathrm{y}$, en general, mejor diseñadas, que las correspondientes a las empresas no familiares. Por tanto, y de acuerdo con la Hipótesis Porter, las empresas familiares serán más capaces que las no familiares de rentabilizar los recursos dedicados a protección del medio ambiente. Los argumentos planteados llevan al establecimiento de la siguiente hipótesis:

H2: Los beneficios económicos derivados de la inversión medioambiental son mayores en las empresas familiares que en las empresas no familiares.

\section{Descripción de la muestra y de las variables relevantes: primeros resultados}

\subsection{Descripción de la muestra y de las variables relevantes}

El contexto en el que vamos a desarrollar el trabajo es el sector manufacturero español. La muestra utilizada se ha obtenido a partir de la información recogida en la Encuesta sobre Estrategias Empresariales (ESEE). 
Tabla 1

Distribución de la muestra según variables relevantes.

\begin{tabular}{|c|c|c|}
\hline Tamaño muestral & $\begin{array}{c}\mathrm{N} \\
1916\end{array}$ & $\begin{array}{c}\% \\
100 \%\end{array}$ \\
\hline \multicolumn{3}{|l|}{ Empresa Familiar } \\
\hline No Familiar & 1092 & $56.99 \%$ \\
\hline Si Familiar & 824 & $43.01 \%$ \\
\hline \multicolumn{3}{|l|}{ Inversión Me } \\
\hline No Realizan & 1440 & $75.16 \%$ \\
\hline Si Realizan & 476 & $24.84 \%$ \\
\hline \multicolumn{3}{|l|}{ Número de empleados } \\
\hline 200 o menos & 1533 & $80.01 \%$ \\
\hline Más de 200 & 383 & $19.99 \%$ \\
\hline \multicolumn{3}{|l|}{ Sector } \\
\hline Industria cárnica & 74 & $3.86 \%$ \\
\hline Productos alimenticios y tabaco & 209 & $10.91 \%$ \\
\hline Bebidas & 42 & $2.19 \%$ \\
\hline Textiles y confección & 125 & $6.52 \%$ \\
\hline Cuero y calzado & 52 & $2.71 \%$ \\
\hline Industria de la madera & 72 & $3.76 \%$ \\
\hline Industria del papel & 76 & $3.97 \%$ \\
\hline Artes gráficas & 74 & $3.86 \%$ \\
\hline $\begin{array}{l}\text { Industria química y productos } \\
\text { farmacéuticos }\end{array}$ & 131 & $6.84 \%$ \\
\hline Productos de caucho y plástico & 108 & $5.64 \%$ \\
\hline Productos minerales no metálicos & 142 & $7.41 \%$ \\
\hline Metales férreos y no férreos & 69 & $3.60 \%$ \\
\hline Productos metálicos & 240 & $12.53 \%$ \\
\hline Máquinas agrícolas e industriales & 108 & $5.64 \%$ \\
\hline $\begin{array}{l}\text { Productos informáticos, } \\
\text { electrónicos y ópticos }\end{array}$ & 31 & $1.62 \%$ \\
\hline Maquinaria y material eléctrico & 79 & $4.12 \%$ \\
\hline Vehículos de motor & 90 & $4.70 \%$ \\
\hline Otro material de transporte & 41 & $2.14 \%$ \\
\hline Industria del mueble & 100 & $5.22 \%$ \\
\hline Otras industrias manufactureras & 53 & $2.77 \%$ \\
\hline
\end{tabular}

En función de los datos proporcionados por la ESEE, se ha construido una muestra de 1916 empresas, para el año 2010. Aunque lo ideal hubiera sido disponer de una serie de datos históricos sobre los que estudiar las hipótesis planteadas, una de las variables relevantes para nuestro análisis, la inversión medioambiental, es un dato recientemente proporcionado por la encuesta, y por este motivo hemos centrado el estudio en el año 2010. Las empresas de la muestra fueron seleccionadas extrayendo de la base de datos ESEE todas las empresas para las que se disponía de información en todas las variables relevantes para el estudio.

Las variables consideradas en el estudio son:
Inversión medioambiental (INV-ME): variable que indica si la empresa realizó inversiones en equipos e instalaciones relacionadas con el control de la contaminación medioambiental. De acuerdo con la información disponible en la encuesta, se mide como una variable dummy que toma valor 1 cuando la empresa realiza inversión medioambiental en el año.

Empresa familiar versus empresa no familiar (FAM): variable que indica si un grupo familiar participa activamente en el control o gestión de la empresa. De acuerdo con la información disponible en la encuesta, se mide como una variable dummy que toma valor 1 cuando la empresa es de tipo familiar.

Rentabilidad Económica (ROA): variable que indica la rentabilidad del capital invertido en activo. $\mathrm{Se}$ ha medido como la ratio beneficio antes de intereses e impuestos dividido por el activo total, aproximados a partir de la información disponible en la ESEE.

Actividad/Sector: han sido consideradas variables sectoriales de control representativas de la actividad principal de la empresa, según agregación de los códigos de 3 dígitos CNAE-09, identificando 20 sectores manufactureros, tal y como se puede ver en la tabla 1.

Tamaño: variable de control que considera dos categorías de tamaño, para distinguir entre empresas con hasta 200 trabajadores y empresas de más de 200 trabajadores.

En la tabla 1 se detalla la distribución de las empresas de la muestra en función de las variables relevantes. En la tabla 2 se detalla la distribución según pares de variables relevantes. Por último, la tabla 3 permite analizar los valores medios de la rentabilidad económica (ROA) por categorías de variables. De los datos presentados en las tablas 1, 2 y 3 se obtienen unos primeros resultados que son comentados en la siguiente subsección.

\subsection{Primeros resultados}

Según se desprende de los datos de la tabla 1, de las 1916 empresas que componen la muestra, el $43,01 \%$ de las mismas son de carácter familiar y el $56,99 \%$ restante son empresas no familiares. Por otra parte, si atendemos a la variable inversión medioambiental se observa que solamente el $24,84 \%$ de las empresas de la muestra realizan inversión medioambiental. 
Tabla 2

Distribución de la muestra según pares de variables.

\begin{tabular}{|c|c|c|c|c|c|}
\hline & Inver & ión $M$ & oambienta & & \\
\hline & No & $\mathrm{Si}$ & $\% \mathrm{Si}$ inv. & & \\
\hline Muestra & 1440 & 476 & $24.8 \%$ & & \\
\hline Empresa Familiar & & & & & \\
\hline No & 798 & 294 & $26.9 \%$ & Empre & Imiliar \\
\hline $\mathrm{Si}$ & 642 & 182 & $22.1 \%$ & $\mathrm{NO}$ & SI \\
\hline Número de empleados & No & $\mathrm{Si}$ & $\% \mathrm{Si}$ inv. & $\%$ Si inv. & $\% \mathrm{Si}$ inv \\
\hline 200 o menos & 1281 & 252 & $16.4 \%$ & $16.0 \%$ & $16.9 \%$ \\
\hline Mas de 200 & 159 & 224 & $58.5 \%$ & $60.4 \%$ & $53.9 \%$ \\
\hline Sector & No & $\mathrm{Si}$ & \% Si inv. & & \\
\hline Industria cárnica & 51 & 23 & $31.1 \%$ & $31.0 \%$ & $31.3 \%$ \\
\hline Productos alimenticios y tabaco & 148 & 61 & $29.2 \%$ & $27.5 \%$ & $30.8 \%$ \\
\hline Bebidas & 24 & 18 & $42.9 \%$ & $33.3 \%$ & $55.6 \%$ \\
\hline Textiles y confección & 117 & 8 & $6.4 \%$ & $8.5 \%$ & $4.5 \%$ \\
\hline Cuero y calzado & 49 & 3 & $5.8 \%$ & $8.8 \%$ & $0.0 \%$ \\
\hline Industria de la madera & 56 & 16 & $22.2 \%$ & $32.4 \%$ & $11.4 \%$ \\
\hline Industria del papel & 55 & 21 & $27.6 \%$ & $29.5 \%$ & $25.0 \%$ \\
\hline Artes gráficas & 64 & 10 & $13.5 \%$ & $14.6 \%$ & $11.5 \%$ \\
\hline Ind. química y productos farmacéuticos & 58 & 73 & $55.7 \%$ & $55.3 \%$ & $56.5 \%$ \\
\hline Productos de caucho y plástico & 84 & 24 & $22.2 \%$ & $30.2 \%$ & $14.5 \%$ \\
\hline Productos minerales no metálicos & 103 & 39 & $27.5 \%$ & $30.7 \%$ & $23.9 \%$ \\
\hline Metales férreos y no férreos & 41 & 28 & $40.6 \%$ & $34.9 \%$ & $50.0 \%$ \\
\hline Productos metálicos & 202 & 38 & $15.8 \%$ & $16.2 \%$ & $15.3 \%$ \\
\hline Máquinas agrícolas e industriales & 83 & 25 & $23.1 \%$ & $22.6 \%$ & $23.9 \%$ \\
\hline Prodtos. informátic., electrón. y ópticos & 23 & 8 & $25.8 \%$ & $23.8 \%$ & $30.0 \%$ \\
\hline Maquinaria y material eléctrico & 60 & 19 & $24.1 \%$ & $30.6 \%$ & $13.3 \%$ \\
\hline Vehículos de motor & 54 & 36 & $40.0 \%$ & $50.0 \%$ & $17.9 \%$ \\
\hline Otro material de transporte & 32 & 9 & $22.0 \%$ & $25.0 \%$ & $11.1 \%$ \\
\hline Industria del mueble & 88 & 12 & $12.0 \%$ & $13.0 \%$ & $10.9 \%$ \\
\hline Otras industrias manufactureras & 48 & 5 & $9.4 \%$ & $4.2 \%$ & $13.8 \%$ \\
\hline
\end{tabular}

Atendiendo a los porcentajes de empresas que realizan inversión medioambiental dentro de las categorías familiar y no familiar, en la tabla 2 se observa que el porcentaje es ligeramente superior en las empresas de tipo no familiar. Así, el 26,9\% de las empresas no familiares invierten en protección medioambiental, mientras que en las empresas familiares las empresas que invierten representan el $22,1 \%$. Este resultado parece indicar que existen diferencias en el comportamiento medioambiental entre las empresas de tipo familiar y las de tipo no familiar. Más concretamente, los primeros resultados del estudio indican que las empresas familiares de la muestra tienden a invertir en protección medioambiental algo menos que las no familiares. Sin embargo, este resultado podría ser justificado más por el menor tamaño medio de las empresas familiares de la muestra, que por una supuesta menor proactividad medioambiental en este tipo de empresas.
En efecto, si atendemos al tamaño de las empresas, en la tabla 1 observamos que el $80 \%$ de las empresas de la muestra tienen menos de 200 trabajadores. Dentro de este grupo de pequeñas empresas, solamente el 16,4\% realizan inversión medioambiental (véase la tabla 2), no existiendo una acusada diferencia en este aspecto entre las familiares y las no familiares. Así, cuando analizamos la propensión inversora dentro de las pequeñas empresas, se observa un pequeño sesgo hacia las empresas de tipo familiar. En concreto, se observa que realizan inversión el $16,9 \%$ de las familiares mientras que entre las no familiares realizan inversión el $16 \%$. En lo que respecta a las empresas grandes (más de 200 trabajadores), suponen el 19,99\% de la muestra (tabla 1). Al analizar su propensión a invertir en protección medioambiental, se aprecia que es mucho mayor que en las pequeñas empresas. En concreto, los datos presentados en la tabla 2 permiten apreciar que un $58,5 \%$ de las empresas grandes invierte en equipos e 
instalaciones de protección medioambiental. Este porcentaje disminuye hasta un $53,9 \%$ cuando nos referimos a empresas familiares, mientras que aumenta hasta un $60,4 \%$ cuando nos referimos a empresas no familiares. Los resultados presentados nos permiten deducir que las grandes empresas familiares muestran una menor propensión que las no familiares hacia la inversión en protección del medio ambiente.

Tal y como se aprecia en la tabla 1, las empresas analizadas se distribuyen en veinte sectores, de los cuales el que cuenta con una mayor presencia en la muestra es el sector de Productos metálicos, con una presencia del 12,53\%, seguido del sector de

Tabla 3

ROA por categorías de variables.

\begin{tabular}{|c|c|c|c|}
\hline & $\bar{x}$ & t-test & ANOVA \\
\hline ROA & 8.05 & & \\
\hline Empresa Familiar & & 0.95 & \\
\hline No Familiar & 8.35 & & \\
\hline Si Familiar & 7.65 & & \\
\hline Inversión Me & & $-4.40 * * *$ & \\
\hline No Realizan & 7.13 & & \\
\hline Sí Realizan & 10.81 & & \\
\hline Tamaño & & $-3.28 * * *$ & \\
\hline 200 o menos & 7.45 & & \\
\hline Más de 200 & 10.43 & & \\
\hline Familiar / Inversión Me & & & $6.90^{* * *}$ \\
\hline No Familiar /No Realizan & 7.54 & & \\
\hline Si Familiar / No Realizan & 6.62 & & \\
\hline No Familiar /Sí Realizan & 10.53 & & \\
\hline Si Familiar / Sí Realizan & 11.26 & & \\
\hline Familiar / Tamaño & & & $6.90^{* * *}$ \\
\hline No Familiar /200 o menos & 7.63 & & \\
\hline Si Familiar / 200 o menos & 7.24 & & \\
\hline No Familiar /Más de 200 & 10.56 & & \\
\hline Si Familiar / Más de 200 & 10.14 & & \\
\hline Sector & & & $3.95^{* * *}$ \\
\hline Industria cárnica & 8.26 & & \\
\hline Productos alimenticios y tabaco & 11.42 & & \\
\hline Bebidas & 12.57 & & \\
\hline Textiles y confección & 6.15 & & \\
\hline Cuero y calzado & 8.83 & & \\
\hline Industria de la madera & 6.53 & & \\
\hline Industria del papel & 12.84 & & \\
\hline Artes gráficas & 8.54 & & \\
\hline Industria química y productos farmacéuticos & 10.62 & & \\
\hline Productos de caucho y plástico & 7.97 & & \\
\hline Productos minerales no metálicos & 2.61 & & \\
\hline Metales férreos y no férreos & 6.82 & & \\
\hline Productos metálicos & 6.76 & & \\
\hline Máquinas agrícolas e industriales & 7.17 & & \\
\hline Productos informáticos, electrónicos y ópticos & 6.90 & & \\
\hline Maquinaria y material eléctrico & 5.12 & & \\
\hline Vehículos de motor & 14.62 & & \\
\hline Otro material de transporte & 10.02 & & \\
\hline Industria del mueble & 3.16 & & \\
\hline Otras industrias manufactureras & 8.17 & & \\
\hline
\end{tabular}


Productos alimenticios y tabaco (10,91\%). Atendiendo a los datos presentados en la tabla 2 se aprecia que, de entre los sectores que tienen mayor propensión a la inversión medioambiental, destaca el sector de Industria química y productos farmacéuticos, donde el $55,7 \%$ de las empresas invierten, no existiendo mucha diferencia en la propensión inversora entre las empresas familiares y las no familiares (el 55,3\% de las empresas no familiares y el $56,5 \%$ de las empresas familiares realizan inversión medioambiental). El siguiente sector en propensión inversora en medio ambiente es el de Bebidas, donde un 42,9\% de las empresas En este sector hay mayores diferencias invierte cuando se atiende a la variable que nos indica si la empresa es o no familiar, ya que mientras invierten el 33,3\% de las empresas no familiares, el porcentaje asciende al $55,6 \%$ cuando las empresas son familiares.

Atendiendo a los sectores en los que la inversión medioambiental es menos probable cabe destacar el sector de Cuero y calzado, en el que solamente invierten en protección del medioambiente un 5,8\% de todas las empresas, existiendo grandes diferencias en la propensión a invertir si diferenciamos por propiedad (el $8,8 \%$ de las empresas no familiares invierten, mientras que el porcentaje de empresas familiares que invierte es el $0 \%$ ). Otro sector destacado por su escaso porcentaje de empresas que invierten en medio ambiente es el de Textiles y confección, donde solamente un $6,4 \%$ de las empresas invierten. Las diferencias según la empresa es o no familiar son también acusadas en este sector (invierten un $8,5 \%$ de las empresas no familiares y un $4,5 \%$ de las empresas familiares).

Los resultados presentados parecen indicar que el sector de actividad resulta relevante cuando se compara la propensión inversora de las empresas según su propiedad sea o no de tipo familiar. Así, mientras que en algunos sectores como el de Industria cárnica o el de Productos metálicos no se aprecian diferencias, en otros las diferencias son muy acusadas. Uno de los sectores destacados en este sentido es el de Vehículos de motor. En este sector el $50 \%$ de las empresas no familiares realizan inversión medioambiental y, sin embargo, solamente un $17,9 \%$ de las empresas familiares presentan este comportamiento.

En la tabla 3 se presentan los resultados de seis Análisis de la Varianza realizados para estudiar las diferencias existentes en la Rentabilidad económica de diferentes tipos de empresa. Los resultados indican que, mientras que la rentabilidad económica

Tabla 4: Resultados de la regresión.

Resultados de la regresión: muestra completa y submuestras según el tipo de propiedad.

\begin{tabular}{|c|c|c|c|c|c|c|}
\hline \multirow[b]{3}{*}{ Constante } & \multicolumn{2}{|l|}{ Muestra } & \multicolumn{2}{|c|}{$\begin{array}{l}\text { Empresa Familiar } \\
\text { Si }\end{array}$} & \multicolumn{2}{|l|}{ No } \\
\hline & Coef. & t-test & Coef.F $_{\text {F }}$ & t-test & $\overline{\text { Coef. }_{\mathrm{NF}}}$ & t-test \\
\hline & 1,805 & 1,352 & 0,407 & 0,241 & 3,030 & 1,514 \\
\hline Inv-me & $2,915 * * *$ & 3,369 & $3,822 * * *$ & 3,145 & $2,358^{*}$ & 1,939 \\
\hline \multicolumn{7}{|l|}{ Sector } \\
\hline Industria cárnica & $5,552 * *$ & 2,475 & $7,200 * *$ & 2,459 & 4,093 & 1,247 \\
\hline Productos alimenticios y tabaco & $8,765 * * *$ & 5,150 & $10,559 * * *$ & 4,972 & $6,983 * * *$ & 2,695 \\
\hline Bebidas & $9,515 * * *$ & 3,458 & $7,172 * *$ & 1,972 & $10,902 * * *$ & 2,730 \\
\hline Textiles y confección & $4,163 * *$ & 2,159 & 3,662 & 1,543 & $5,062 *$ & 1,701 \\
\hline Cuero y calzado & $6,856 * * *$ & 2,696 & $8,647 * *$ & 2,384 & 5,471 & 1,550 \\
\hline Industria de la madera & $4,076^{*}$ & 1,800 & $5,093 *$ & 1,790 & 3,293 & 0,962 \\
\hline Industria del papel & $10,227 * * *$ & 4,598 & $11,142 * * *$ & 3,807 & $9,352 * * *$ & 2,892 \\
\hline Artes gráficas & $6,339 * * *$ & 2,821 & $7,855 * *$ & 2,493 & 5,074 & 1,609 \\
\hline Industria química y productos farmacéuticos & $7,195 * * *$ & 3,764 & $6,878 * * *$ & 2,607 & $6,928 * *$ & 2,552 \\
\hline Productos de caucho y plástico & $5,522 * * *$ & 2,763 & $8,682 * * *$ & 3,500 & 2,498 & 0,817 \\
\hline Metales férreos y no férreos & $3,828^{*}$ & 1,665 & $8,335 * * *$ & 2,635 &, 642 & 0,197 \\
\hline Productos metálicos & $4,493 * * *$ & 2,707 & $5,803 * * *$ & 2,685 & 3,323 & 1,363 \\
\hline Máquinas agrícolas e industriales & $4,688 * *$ & 2,346 & $4,860 *$ & 1,863 & 4,336 & 1,483 \\
\hline Productos informáticos, electrónicos y ópticos & 4,342 & 1,400 & $11,590 * *$ & 2,510 & ,334 & 0,079 \\
\hline Maquinaria y material eléctrico & 2,612 & 1,189 &, 111 & 0,037 & 3,869 & 1,237 \\
\hline Vehículos de motor & $11,649 * * *$ & 5,518 & $15,033 * * *$ & 4,904 & $9,732 * * *$ & 3,318 \\
\hline Otro material de transporte & $7,571 * * *$ & 2,729 & 7,408 & 1,531 & $6,895^{*}$ & 1,917 \\
\hline Industria del mueble & 1,002 & 0,489 & 2,346 & 0,898 &,- 190 &,- 062 \\
\hline Otras industrias manufactureras & $6,089 * *$ & 2,413 & 4,327 & 1,428 & $8,552 * *$ & 2,134 \\
\hline $\mathrm{N}^{\mathrm{o}}$ de observaciones & 1916 & & 824 & & 1092 & \\
\hline
\end{tabular}


es similar en las empresas familiares y no familiares, sí existen diferencias significativas según el tamaño de la empresa y según se realiza o no inversión medioambiental, resultando significativamente más rentables las empresas de más de 200 empleados y las que realizan inversión en protección del medio ambiente. La condición de familiar o no familiar sí parece afectar a la rentabilidad económica cuando se combina con las variables inversión medioambiental y tamaño. Por último, en la tabla 3 se aprecian también diferencias significativas en la rentabilidad económica media de los diferentes sectores. Estos resultados ponen de manifiesto la necesidad de considerar variables de control en los análisis dirigidos a contrastar las hipótesis planteadas.

\section{Metodología y resultados del contraste de hipótesis}

\subsection{Metodología}

La metodología que se ha utilizado para contrastar las hipótesis ha sido la regresión lineal. El modelo teórico planteado trata de medir el impacto de la variable inversión medioamb iental en los resultados de las empresas, medidos mediante la Rentabilidad económica (ROA). Adicionalmente, para corregir el efecto sectorial en los resultados, se incluyen variables de control para considerar el sector de actividad, a través de variables dummy (tomando como sector de referencia el que presenta un menor ROA, que es el de Productos minerales no metálicos, según se puede observar en la tabla 3 ).

Con respecto a la variable tamaño, no se incluye en las regresiones como variable de control por presentar cierta correlación con la variable inversión medioambiental. Sin embargo, y con el objetivo de observar la influencia del tamaño en las relaciones entre las variables, las regresiones son también estimadas para diferentes grupos de tamaño.

Concretamente se realizan siete regresiones para contrastar el mismo modelo teórico, pero en siete muestras diferentes: la muestra completa, la submuestra compuesta por las empresas familiares, la submuestra compuesta por las empresas no familiares, y las cuatro submuestras que combinan las categorías empresa familiar o empresa no familiar con las dos categorías de tamaño (hasta 200 o más de 200 empleados).

\subsection{Resultados de los contrastes de hipótesis}

Los resultados presentados en la tabla 4 son los relativos a la regresión realizada para toda la muestra y a las regresiones realizadas para las muestras de empresas familiares y de empresas no familiares. Los resultados de las regresiones realizadas para las submuestras que combinan el tipo de propiedad con el tamaño se presentan en la tabla 5.

En la muestra global de las 1916 empresas manufactureras españolas, el impacto de la inversión medioambiental tiene un efecto positivo en los resultados financieros. Este resultado, que se mantiene tanto en la submuestra de empresas familiares como en la de no familiares, es coherente con la idea de que implicarse en proyectos de protección medioambiental permite el aprovechamiento de ciertas ventajas, que conducirán a las empresas hacia la mejora de sus resultados económicos. En el contexto español, autores como Brío y Junquera (2004), por ejemplo, comprueban que algunas de las ventajas derivadas de emprender acciones de tipo medioambiental son la mejora de la imagen corporativa, la mejora de las relaciones con los stakeholders, la mejora en las condiciones de trabajo y en la moral de los empleados, y la mejora de las posibilidades de entrada en mercados internacionales.

Sin embargo, tal y como se aprecia en la tabla 5 , el hecho de invertir en protección medioambiental no tiene un efecto significativo en el ROA de las empresas de más de 200 empleados (tanto en la submuestra de empresas familiares como en la de no familiares). La Hipótesis 1 resulta, por tanto, parcialmente soportada. La obtención de una situación win-win en la que la inversión medioambiental permite mejorar tanto los resultados medioambientales como los económicos no puede darse por garantizada, sino que parece depender de las condiciones en las que la inversión es realizada. La idea de que existen diferentes formas de implementación de los proyectos de inversión medioambiental está muy presente en la literatura. En referencia al contexto español, ya algunos autores como González-Benito y González-Benito (2005b) constatan, a partir de un estudio realizado en tres sectores manufactureros españoles, que existen diferentes perfiles de proactividad medioambiental en la conducta de las empresas. Amores-Salvadó et al. (2012), por su parte, establecen que el paso de conductas de control de la contaminación hacia conductas más avanzadas de prevención de la contaminación en las empresas españolas, está relacionado con otros aspectos, al encontrar relevante en la consecución de este paso el hecho de 
Tabla 5

Resultados de la regresión: submuestras combinación del tipo de propiedad y el tamaño.

\section{Empresas de hasta 200 trabajadores Empresas de más de 200}

Familiar No Familiar $\quad$ Familiar $\quad$ No Familiar

\begin{tabular}{|c|c|c|c|c|c|c|c|c|}
\hline & Coef.F $_{\text {F }}$ & $\begin{array}{l}\text { t- } \\
\text { test }\end{array}$ & Coef. $_{\mathrm{NF}}$ & t-test & Coef.F $_{\text {. }}$ & t-test & Coef. $_{\mathrm{NF}}$ & t-test \\
\hline Constante & 0,177 & 0,10 & 2,205 & 0,98 & 3,639 & 0,80 & 7,312 & 1,61 \\
\hline $\begin{array}{l}\text { Inv-me } \\
\text { Sector }\end{array}$ & $3,858 * * *$ & 2,61 & $3,002^{*}$ & 1,82 & 2,376 & 1,09 & $-0,061$ & $-0,03$ \\
\hline Industria cárnica & $7,398^{* *}$ & 2,26 & 5,041 & 1,38 & 5,524 & 0,90 & $-0,231$ & $-0,03$ \\
\hline Productos alimenticios y tabaco & $9,288 * * *$ & 3,99 & $6,195^{* *}$ & 2,06 & $14,757 * *$ & 3,00 & 7,191 & 1,38 \\
\hline Bebidas & $8,654 * *$ & 2,14 & $11,731 * *$ & 2,39 & $-1,451$ & $-0,19$ & 7,738 & 1,09 \\
\hline Textiles y confección & 3,047 & 1,20 & $5,829^{*}$ & 1,83 & 11,015 & 1,70 & 1,525 & 0,16 \\
\hline Cuero y calzado & $8,877 * *$ & 2,36 & 6,238 & 1,70 & - & - & - & - \\
\hline Industria de la madera & $6,155^{*}$ & 2,04 & 4,349 & 1,19 & $-11,209$ & $-1,30$ & $-4,161$ & $-0,38$ \\
\hline Industria del papel & $11,333 * * *$ & 3,56 & $11,561 * * *$ & 3,00 & 8,846 & 1,30 & 3,056 & 0,5 \\
\hline Artes gráficas & $8,080 * *$ & 2,47 & $6,094^{*}$ & 1,82 & - & , & $-2,914$ & $-0,23$ \\
\hline $\begin{array}{l}\text { Industria química y productos } \\
\text { farmacéuticos }\end{array}$ & $6,424^{* *}$ & 2,08 & $7,309^{* *}$ & 2,29 & 6,095 & 1,18 & 5,408 & 1,03 \\
\hline Productos de caucho y plástico & $9,495 * * *$ & 3,56 & 4,442 & 1,27 & 0,149 & 0,02 & $-3,673$ & $-0,59$ \\
\hline Metales férreos y no férreos & $11,458 * * *$ & 2,98 & 4,921 & 1,20 & 0,573 & 0,10 & $-6,739$ & $-1,17$ \\
\hline Productos metálicos & $6,384 * * *$ & 2,75 & 3,721 & 1,37 & $-0,132$ & $-0,02$ & 2,219 & 0,40 \\
\hline $\begin{array}{l}\text { Máquinas agrícolas e } \\
\text { industriales }\end{array}$ & $5,423^{*}$ & 1,85 & 4,445 & 1,32 & 1,127 & 0,21 & 2,857 & 0,48 \\
\hline $\begin{array}{l}\text { Productos informáticos, } \\
\text { electrónicos y ópticos }\end{array}$ & $13,194 * *$ & 2,65 & $-8,109$ & $-1,56$ & $-4,122$ & $-0,36$ & 12,239 & 1,65 \\
\hline Maquinaria y material eléctrico & $-0,190$ & $\overline{-} 0,06$ & 4,157 & 1,18 & $-0,759$ & $-0,13$ & 2,507 & 0,38 \\
\hline Vehículos de motor & $14,879^{* * *}$ & 4,29 & $7,301 *$ & 1,77 & $13,424 * *$ & 2,17 & $8,913^{*}$ & 1,77 \\
\hline Otro material de transporte & 3,858 & 0,74 & $11,548 * *$ & 2,53 & $34,378 * *$ & 2,99 & $-1,146$ & $-0,18$ \\
\hline Industria del mueble & 2,681 & 0,94 & 0,533 & 0,16 & $-1,374$ & $-0,22$ & $-2,227$ & $-0,21$ \\
\hline Otras industrias manufactureras & 4,315 & 1,35 & $8,702 * *$ & 2,05 & 9,191 & 0,80 & 12,893 & 1 \\
\hline N. de observaciones & 709 & & 824 & & 115 & & 268 & \\
\hline
\end{tabular}

disponer o no de un estándar medioambiental certificado.

De acuerdo con la literatura, la forma en las que las acciones de protección medioambiental son implementadas condiciona la posible obtención de beneficios económicos derivados de tales acciones (King y Lenox, 2001; 2002; Guenster et al., 2006). En nuestro estudio, si comparamos los resultados de las dos regresiones presentadas en la tabla 4 para las submuestras de empresas familiares y no familiares, se observa que el impacto de la inversión medioambiental en los resultados financieros es superior en las empresas familiares. Este resultado se mantiene cuando se comparan las submuestras familiar y no familiar en las dos categorías de tamaño (tabla 5), lo que da soporte a la Hipótesis 2 planteada en el estudio.

Los resultados presentados en la tabla 5, relativos a regresiones estimadas por grupos según el tamaño de las empresas y la propiedad familiar o no de las mismas, permiten obtener resultados adicionales.
Estos resultados indican que las empresas que mejores resultados económicos obtienen de la inversión medioambiental son las empresas familiares con hasta 200 trabajadores. Por el contrario, las empresas donde el impacto económico de la inversión medioambiental es menor son las empresas de más de 200 trabajadores y no familiares.

\section{Conclusiones}

En los últimos años, la mayor regulación en materia de medio ambiente y la mayor preocupación social, han conducido a las empresas hacia la realización de inversiones medioambientales cuyas consecuencias financieras son todavía objeto de debate el ámbito académico y profesional. En este trabajo han sido analizados datos correspondientes a empresas manufactureras españolas, recogidos a través de la encuesta ESEE, con el objetivo de contrastar si el resultado financiero de las empresas 
mejora al realizar inversiones de protección medioambiental y con el objetivo de medir el impacto que tiene el tipo de propiedad (familiar o no familiar) en esa relación.

Del estudio podemos destacar las siguientes conclusiones más relevantes. En primer lugar, se concluye que mientras que en las pequeñas empresas el tipo de propiedad (familiar o no familiar) no influye en la propensión a invertir en protección del medio ambiente, esta condición sí influye cuando las empresas son grandes. En las grandes empresas, la propensión a invertir en protección del medio ambiente es mayor cuando las empresas son de tipo no familiar que cuando un grupo familiar participa activamente en el control o la gestión de la empresa.

En segundo lugar, del análisis de las consecuencias económicas derivadas de invertir en protección del medio ambiente se concluye que la posibilidad de mejorar los beneficios económicos depende de las circunstancias, internas o externas a la empresa, que acompañan a la implementación de los proyectos de mejora medioambiental. Por este motivo, la posibilidad de mejorar los beneficios económicos es mayor en las pequeñas que en las grandes empresas y también es mayor en las empresas de tipo familiar que en las de tipo no familiar.

Analizado el efecto de la inversión medioambiental en los resultados económicos de cuatro submuestras de empresas manufactureras, según su tamaño e influencia de un grupo familiar en su control, se concluye que son las pequeñas empresas de tipo familiar las más capaces de alcanzar una situación win-win en la que la mejora de los resultados medioambientales puede venir acompañada de una mejora en los resultados económicos.

Dentro de las grandes empresas, el hecho de que sea un grupo familiar el que controle la gestión de la empresa modera también la relación entre la inversión medioambiental y la rentabilidad económica, contribuyendo a la rentabilización de la inversión medioambiental.

En síntesis, las conclusiones obtenidas en este trabajo sugieren que las empresas analizan con mucho más detalle la decisión de invertir en proyectos de protección medioambiental cuando están controladas por un grupo familiar. De hecho, y especialmente cuando tienen una gran dimensión, las empresas familiares acaban implicándose en proyectos verdes en menor medida que las no familiares. Sin embargo, los proyectos de inversión verde escogidos por las empresas familiares parecen estar mejor diseñados y/o implementados que los correspondientes a las empresas no familiares, lo que permite a las primeras la obtención de beneficios económicos relativamente mayores.

Ahora bien, este trabajo presenta una serie de limitaciones que es conveniente reconocer. Primero, la naturaleza del estudio realizado es estática. Sin embargo, el Enfoque de las Capacidades Dinámicas invita a la aplicación de otras metodologías, como el análisis de datos panel, lo que permitiría observar la existencia de efectos dinámicos. Segundo, sería interesante ampliar el número de sectores analizados, así como el ámbito geográfico objeto de estudio, dado que enriquecería notablemente los resultados obtenidos. Tercero, la evidencia de que la relación entre inversión medioambiental y resultado económico es compleja, y depende del efecto moderador de ciertas circunstancias, exige una profunda reflexión sobre muchas otras variables que podrían intervenir en esta relación, además de la forma de gobierno de la empresa. En este sentido, la incorporación de nuevos enfoques teóricos, como la Teoría de los Stakeholders o el Enfoque Cognitivo, puede resultar de gran valor.

Por último, estas conclusiones dejan abiertas nuevas preguntas de investigación en las que sería necesario seguir profundizando. En este sentido, invitan a profundizar en el estudio y conocimiento del saber hacer de las empresas familiares en materia de gestión medioambiental. Por ejemplo, Sharma y Sharma (2011) sugieren que el nivel de conflicto entre quienes ostentan el control de la empresa familiar podría actuar como variable moderadora en la relación entre la intención de emprender estrategias medioambientales proactivas y las acciones adoptadas para el desarrollo de capacidades organizativas valiosas en este sentido. En efecto, conocer la forma y las condiciones en la que los proyectos de inversión medioambiental son implementados en las empresas familiares ayudaría a entender la forma en la que dichos proyectos pueden resultar rentables y no solamente ecológicos. Adicionalmente, las conclusiones de este trabajo invitan a las grandes empresas familiares hacia una mayor propensión a invertir en proyectos de protección medioambiental, que permitirían mejorar sus resultados económicos además de sus resultados medioambientales.

\section{Bibliografía}

Amores-Salvadó, J., Navas-López, J.E., Martín-deCastro, G. y Delgado-Verde, M. (2012). El Paso del Control a la Prevención: ¿Importan los Estándares 
Medioambientales? Universia Business Review, 34, 6881.

Aragón Correa, J.A. y Sharma, S. (2003). A Contingent Resource-Based View of Proactive Corporate Environmental Strategy. Academy of Management Review, 28(1), 71-88.

Berrone, P., Cruz, C., Gomez Mejía, L. y Larraza, M. (2009). Governance and Environmental Performance in Family Controlled Public Corporations. Frontiers of Entrepreneurs Research, 29(14), Article 2.

Bingham, J., Gibb Dyer, W., Smith, I. y Adams, G. (2011). A Stakeholder Identity Orientation Approach to Corporate Social Performance in Family Firms. Journal of Business Ethics, 99, 565-585.

Bosworth, W. y Clemens, B. (2011). Does It Pay to Be Environmentally Responsible?. Toxic Releases and Financial Performance. Journal of Strategic Innovation and Sustainability, 7(2), 115-121.

Brio, J.A. y Junquera, B. (2004). Competitividad y Situación Medioambiental de los Centros Productivos Certificados en España. Universia Business Review, 4, p. 64-77.

Clemens, B. (2006). Economic Incentives and Small Firms: Does it Pay to be Green? Journal of Business Research, 59(4), 492-500.

Cañón de Francia, J. y Garcés Ayerbe, C. (2009). ISO 14001 Environmental Certification: A sign valued by the market? Environmental and Resource Economics, 44, 245-262.

Christmann, P. (2000). Effects of Best Practices of Environmental Management on Cost Advantage: The Role of Complementary Assets. Academy of Management Journal, 43, 663-680.

Clemens, B. y Bakstran, L. (2010). A Framework of Theoretical Lenses and Strategic Purposes to Describe Relationships among Firm Environmental Strategy, Financial Performance, and Environmental Performance. Management Research Review, 33(4), 393-405.

Corona, J y Téllez Roca, J (2011). El Protocolo Familiar en Empresa Familiar: Aspectos Jurídicos y Económicos. Barcelona, España: Ed. Deusto, 75.

Craig, J. y Dibrell, C. (2006). The Natural Environment, Innovation and Firm Performance: A Comparative Study. Family Business Review, XIX(4), 275-288.

Darnall, N., Henriques, I. y Sadorsky, P. (2007). Do Environmental Systems Improve Business Performance in an International Setting? Journal of International Management, 14(4), 364-376.

Filbeck, G. y Gorman, R.F. (2004). The Relationship between the Environmental and Financial Performance of Public Utilities. Environmental and Resource Economics, 29, 137-157.
Gallego, I. (2012). La Empresa Familiar. Su Concepto y Delimitación Jurídica. Cuadernos de Reflexión de la Cátedra Prasa de la Empresa Familiar. Marzo 2012.

González Benito, J. y González Benito, O. (2005a). Environmental Proactivity and Business Performance: An Empirical Analysis. OMEGA The International Journal of Management Science, 33(1), 1-15.

González Benito, J. y González Benito, O. (2005b). Perfiles de Proactividad Medioambiental: Evidencia en Empresas Industriales Españolas. Universia Business Review, 5, 92-101.

Griffin, J.J. y Mahon, J.F. (1997). The corporate social performance and corporate financial performance debate: Twenty-five years of incomparable research. Business and Society, 36, 5-31.

Guenster, N., Derwall, J., Bauer, R. y Koedijk, K. (2006). The Economic Value of Corporate EcoEfficiency. European Financial Management, 17(4), 679704.

Hart, S.L. y Ahuja, G. (1996). Does it Pay to be Green? An Empirical Examination of the Relationship between Emission Reduction and Firm Performance. Business Strategy and the Environment, 5, 30-37.

Hart, S.L. (1995). A Natural Resourced-Based View of the Firm. Academic of Management Review, 20(4), 986-1014.

Huang, Y., Ding, H. y Kao, M. (2009). Salient Stakeholder Voices Family Business And Green Innovation Adoption. Journal of Management and Organization, 15(3), 309-326.

Judge, W.Q. y Douglas. T.J. (1998). Performance Implications of Incorporating Natural Environmental Isues into the Strategic Planning Process: An Empirical Assessment. Journal of Management Studies, 35, 241-62.

King, A. y Lenox, M. (2001). Does it Really Pay to be Green? An Empirical Study of Firm Environmental and Financial Performance. Journal of Industrial Ecology, 5, 105-116.

King, A. y Lenox, M. (2002). Exploring the Locus of Profitable Pollution Reduction. Management Science, 48, 289-299.

Klassen, R. y Whybark, D. (1999). The Impact of Environmental Technologies on Manufacturing Performance. Academy of Management Journal, 42(6), 599-615.

Konar, S. y Cohen, M.A. (2001). Does the Market Value Environmental Performance. Review Economics and Statistics, 83, 281-289.

Lanoie, P., Laurent Lucchetti, J., Johnstone, N. y Ambec, S. (2011). Environmental Policy, Innovation and Performance: New Insights on the Porter Hypothesis. Journal of Economics and Management Strategy, 20(3), 803-842. 
Margolis, J.D. y Walsh, J.P. (2001). Misery Loves Companies: Rethinking Social Initiatives by Business. Admnistrative Science Quarterly 48, 268-305.

Nakamura, E. (2011). Does Environmental Investment Really Contribute to Firm Performance?: An empirical Analysis Using Japanese Firms. Eurasian Business Review, 1(2), 91-111.

Porter, M. y Van der Linde, C. (1995). Green and Competitive. Harvard Business Review, 73(5), 120-134.

Porter, M. (1991). America's Green Strategy. Scientific American, 4, 68-168.

Russo, M. V. y Fouts, P. A. (1997). A Resource-Based Perspective on Corporate Environmental Performance and Profitability. Academy of Management Journal, 40(3), 534-559.

Sharma, P. y Sharma, S. (2011). Drivers of Proactive Environmental Strategy in Family Firms. Business Ethics Quarterly, 21(2), 309-334.

Sharma, S. y Vredenburg, H. (1998). Proactive Corporate Environmental Strategy and the Development of Competitively Valuable Organizational Capabilities. Strategic Management Journal, 19, 729-753.
Telle, K. (2006). It Pays to be Green: A Premature Conclusion? Environmental and Resource Economics, 35, 195-220.

Uhlaner, L.M., Van Goor-Balk, H. y Masurel, E. (2004). Family Business and Corporate Social Responsibility in a Sample of Dutch Firms. Journal of Small Business and Enterprise Development, 11(2), 186194.

Wagner, M. (2010). Corporate Social Performance and Innovation with High Social Benefits: a Quantitative Analysis. Journal of Business Ethics, 94(4), 581-594.

Walls, J.L., Phan, P. y Berrone, P. (2008). An Assessment of the Construct Validity of Environmental Strategy Measures. Ross School of Business Working Paper Series, núm. 1105.

Xepapadeas A. y Zeeuw A. (1999). Environmental Policy and Competitiveness: The Porter Hypothesis and the Composition of Capital. Journal of Environmental Economics and Management, 37, 165-182.

Ziegler, A., Schröder, M. y Rennings, K. (2007). The Effect of Environmental and Social Performance on the Stock Performance of European Corporations. Environmental Resource and Economics, 37, 661-680. 